TECHNION-PHYS-96-14

hep-ph/9605441

\title{
Regge asymptotics and color suppressed heavy meson decays
}

\author{
Boris Blok and Igor Halperin 0 \\ Technion - Israel Institute of Technology \\ Department of Physics \\ Haifa, 32000, Israel \\ e-mail addresses: phr34bb@vmsa.technion.ac.il \\ higor@techunix.technion.ac.il
}

\begin{abstract}
:
We discuss a possible generation of color suppressed B-decays amplitudes through a soft final state interaction. As a typical example, we consider in detail the decay $\bar{B}^{0} \rightarrow D^{0} \pi^{0}$ (and also $\bar{B}^{0} \rightarrow 2 \pi^{0}$ ). We show that in the approximation of the two particle unitarity and at zero order in $\alpha_{s}$ this process can be related to the weak decay $\bar{B}^{0} \rightarrow D^{+} \pi^{-}$followed by the strong charge exchange scattering in the Regge kinematics. We estimate the amplitude of this process using the light cone QCD sum rule technique and find that it is supppressed as a power of $1 / m_{B}$ in comparison to the amplitude generated by the effective non-leptonic Hamiltonian, but remains important for the physical value of $m_{B}$
\end{abstract}

submitted to Physics Letters B

* Address after October 1, 1996 : Physics Department, University of British Columbia, 6224 Agriculture Road, Vancouver, BC V6T 1Z1, Canada 


\section{Introduction}

Understanding final state interactions (FSI) effects in weak decays is a challenging task of acute interest, both theoretical and phenomenological. Among plenty of problems, strongly influenced by possible FSI, one can mention a determination of the Kabayashi-Maskawa matrix elements, weak $\mathrm{CP}$ violating phases, etc. It is tremendously difficult to address the issue of FSI on a quantitative level. Still, one may believe that with going to heavier $(c, b)$ quarks possible FSI would become less and less important. In most intuitively appealing form this expectation has been expressed by Bjorken [1], who viewed this behavior as a typical manifestation of the color transparency phenomenon [2]. This scenario can be explained as follows. When a virtual W-boson decays into a $u \bar{d}$ pair with a low invariant mass, this pair is initiallly only weakly interacting with the bulk of the system as its color dipole moment is small. On the other hand, the pair escapes far away from the rest of decay products before starts to interact strongly, as a result of the relativistic time dilation. It follows on these grounds that in decays like $B \rightarrow D \pi$ FSI die off asymptotically as $m_{b} \rightarrow \infty$. In effect, weak non-leptonic decay amplitudes factorize into a product of hadron matrix elements; hard gluons, which otherwise would violate this factorization, do not manage to appear on time.

It is worth noticing that this scenario, while is very attractive in view of its simplicity and appeals to the one's intuition, is only qualitative and does not predict the rate of vanishing FSI corrections when $m_{b} \rightarrow \infty$. Moreover, by its very appearence, this argumentation is likely to be applicable only to FSI proceeding via a hard gluons exchange. In fact, the factorization property has been proved to all orders of the perturbation theory in the heavy quark limit [3] $m_{c}, m_{b} \rightarrow \infty, m_{c} / m_{b}$ fixed. On the other hand, in the case of non-perturbative soft FSI the time dilation is not so important, and one may expect that those effects could be sizeable in spite of the large b-quark mass. Moreover, if initial wave packages hadronize without gluon FSI when their separation is not yet large $(\sim 0.3 \mathrm{fm})$, then there usual hadron-hadron scattering would be a dominant mechanism of FSI. If this kind of FSI is strong enough, it presumably could screen the effect of absence of perturbative FSI, which is due to the color transparency phenomenon. The present paper is just an attempt to provide a semi-quantitative estimate of a possible role of hadronic FSI corrections in non-leptonic B-decays of the type $B \rightarrow D \pi$. Our approach is essentially based on the existence of large parameter in the problem $\left(m_{B}\right)$, and differs from more traditional approaches to FSI in weak decays.

We would like to start our presentation by explaining why FSI corrections are so elusive and hard to treat in most non-perturbative methods which have been applied to non-leptonic B-decays. As has been first explained in Ref. [4], the problem lies

in the fact that physical amplitudes are initially extrapolated into the Euclidean region where a calculation is feasible, and then are analytically continued back into the Minkowsky space-time (this is the typical strategy e.g. in the QCD sum rule 
method [5] or lattice calculations). Atter the Wick rotation fastly oscillating pole terms (corresponding to particles interacting in the final state) become falling ones and die off in the deep Euclidean region. On the other hand, when the amplitude is restored from a dispersion relation, one integrates actually over some finite region of the invariant mass where these oscillating terms are averaged to some smooth function. In effect, amplitudes are always real within these methods; scattering phases cannot be calculated. An analogous statement in the lattice approach has later become known as the Maiani-Testa no-go theorem [6, 7].

In this paper we try to estimate semi-quantitatively FSI corrections to the Bdecays of the form $B \rightarrow D \pi$. We consider the limit of the heavy b-quark. As for the c-quark, we distinguish between two different limits: (i) c-quark is heavy with $m_{b} \gg m_{c}$ and (ii) c-quark is light, the corresponding process is $B \rightarrow \pi \pi$. To simplify the problem and avoid complications due to presence of many channels, we have chosen to look for FSI effects on color suppressed B-decays (more specifically, we consider a particular case of the $\bar{B}^{0} \rightarrow D^{0} \pi^{0}$ decay) and work at zero order in $\alpha_{s}$. Our approach is strongly correlated with the fact that the energy excess in B-decays of this type is large which, as we show, imposes a relevance of the Regge approach. Surprisingly, we find that though FSI effects are suppressed as a power of $1 / m_{B}$ in comparison with direct amplitudes, they remain to be of the same order of magnitude at the physical value $m_{B}=5.27 \mathrm{GeV}$. Here we have to mention a recent paper [8] where similar to ours ideas have been applied to another class of B-decays. We still believe that we manage to reach more concrete motivations and results for our particular decay mode. We will compare our conclusions to those of Ref. [8] in the last section of this paper. In Sect.2 we show that in the approximation of two particle unitarity the decay of interest can be related to a process of strong charge exchange scattering in the Regge kinematics and calculate a discontinuity of a corresponding Feynman diagram. In Sect. 3 and 4 we apply the light cone QCD sum rule technique [9] (see e.g. [10] for a mini-review) to estimate parameters of interest. As a by-product of our study, we derive new light cone sum rules for the $B \rightarrow D$ transition form factor (Sect.3) and $\rho D$ coupling constant $g_{\rho D D}$ (Sect.4) . In Sect.5 we compare the calculated reggeon-induced amplitude to that proceeding directly via corresponding terms in the effective non-leptonic Hamiltonian. Our result is consistent with the color trasparency picture as long as the former is suppressed approximately as $1 / m_{B}$ in respect to the latter. However, we find that FSI corrections are pretty large for the physical value $m_{B} \simeq 5.27 \mathrm{GeV}$ and constitute $40-100 \%$ of the amplitude of direct process. Sect.6 contains a discussion of our results. 


\section{Discontinuity of the $\bar{B} \rightarrow D^{0} \pi^{0}$ amplitude}

We consider the following simplified model for the process of interest. Since at the zero order in $\alpha_{s}$ the direct decay $\bar{B} \rightarrow D^{0} \pi^{0}$ does not occur, we seek for a hadronic intermediate state which would proceed non-perturbatively into the given final state. The most obvious candidate is the $D^{+} \pi^{-}$intermediate state which is related to $D^{0} \pi^{0}$ by the quark rearrangement. In what follows we restrict ourselves by this particular contribution only, i.e. work in the approximation of two particle unitarity relation. Corrections due to multiparticle intermediate states of the form $D^{+}+n \pi$ are presumably small, though accurate estimates are troublesome. Still, calculations within the quark gluon string model [11] indicate that the averaged pion multiplicity is low (2-3), and thus we hope that the two particle approximation is satisfactory with an accuracy not worse than the factor two.

The discontinuity of the amplitude is given by the expression

$$
\operatorname{Disc} M\left(k^{2}\right)=\int \frac{d^{4} p}{(2 \pi)^{2}} \delta\left((p-k)^{2}\right) \delta\left(p^{2}-m_{D}^{2}\right) T\left(k^{2},(k-l-p)^{2}\right) A_{W}\left(B \rightarrow D^{+} \pi^{-}\right)
$$

where $T\left(k^{2},(k-l-p)^{2}\right)$ is the strong charge exchange amplitude for the process $D^{+}(p)+\pi^{-}(k-p) \rightarrow D^{0}(k-l)+\pi^{0}(l)$ and $A_{W}(B \rightarrow D \pi)$ is the weak amplitude for the transition $\bar{B}^{0} \rightarrow D^{+}(p)+\pi^{-}(k-p)$. Using the bare weak Hamiltonian

$$
H_{W}=\frac{G_{F}}{\sqrt{2}} V_{c b} V_{u d}^{*}\left(\bar{c} \Gamma_{\mu} b\right)\left(\bar{d} \Gamma_{\mu} u\right)
$$

$\left(\Gamma_{\mu}=\gamma_{\mu}\left(1-\gamma_{5}\right)\right)$ and taking into account delta functions in the above formula, we obtain

$$
A_{W}(B(k) \rightarrow D(p) \pi(k-p))=i f_{\pi} \frac{G_{F}}{\sqrt{2}} V_{c b} V_{u d}^{*}\left(k^{2}-m_{D}^{2}\right) f_{+}\left(0, k^{2}\right)
$$

where the form factor $f_{+}\left(0, k^{2}\right)$ is defined according to

$$
\left\langle D(p)\left|\bar{c} \Gamma_{\mu} b\right| B(k)\right\rangle=f_{+}\left((k-p)^{2}, k^{2}\right)\left(k_{\mu}+p_{\mu}\right)+f_{-}\left((k-p)^{2}, k^{2}\right)\left(k_{\mu}-p_{\mu}\right)
$$

Here we have introduced an additional variable $k^{2}$ into the standard definition of the $B \rightarrow D$ form factor bearing in mind a subsequent analytical continuation in $k^{2}$. We note that on the mass shell the form factor $f_{+}\left(0, m_{B}^{2}\right)$ can be expressed via the Isgur-Wise function 12

$$
f_{+}\left(0, m_{B}\right)=\frac{m_{B}+m_{D}}{\sqrt{4 m_{B} m_{D}}} \xi\left(1+\frac{\left(m_{B}-m_{D}\right)^{2}}{2 m_{B} m_{D}}\right)
$$

We will not use, however, this correspondence but rather derive a new light cone sum rule which is more suitable for an analytical continuation and refers directly 
to $f_{+}\left(0, m_{B}^{2}\right)$. We delay a corresponding calculation until the next section and concentrate first on the structure of the integral (1). An integration of Eq.(1) yields

$$
\operatorname{Disc} M(s)=\frac{i}{4 \pi} f_{\pi} \frac{G_{F}}{\sqrt{2}} V_{c b} V_{u d}^{*} f_{+}(0, s) \int_{-1}^{1} d(\cos \theta) T(s, t) \frac{\left(s-m_{D}^{2}\right)^{2}}{4 s} \Theta\left(s-m_{D}^{2}\right)
$$

Here $\theta$ is an angle between the momenta $\vec{k}-\vec{l}$ and $\vec{p}$ :

$$
t=(k-l-p)^{2}=-\frac{\left(k^{2}-m_{D}^{2}\right)^{2}}{2 k^{2}}(1-\cos \theta)
$$

The following observation is crucial for the rest of this paper. First, we note that Eq.(6) contains the s-wave partial strong amplitude, in accord with the angular momentum conservation. Therefore, one could think at the first sight that the Regge phenomenology, as being stemming from a resummation of high partial amplitudes, is of no use here. However, it has been known for a long time that partial amplitudes, obtained from Regge amplitudes by extrapolation into a low energy region, reveal resonance structures. This means that a correct answer is to be obtained by first resummation of partial waves into a Regge form, and then re-expanding it back into partial amplitudes. It is useful for a moment to think of the final state interaction as being proceeding through a resonances formation. Anticipating the later result (see Eq.(13) below) we note that the discontinuity in the dispersion integral is a slow (power-like) function of $s$. Therefore in view of a large parameter $m_{B}^{2}$ in the denominator a very large region of $s$ from the threshold $s=\left(m_{D}+m_{\pi}\right)^{2}$ to $s \sim m_{B}^{2}$ contributes in the dispersion integral. It is quite clear that in this parametrically large region many overlapping resonances are created. According to the well known particle - reggeon duality phenomenon s-channel resonances can be be described by a t-channel reggeon exchange [13]. To avoid possible misunderstanding, we should emphasize a difference of our case from the duality picture which applies, strictly speaking, only to an imaginary part of scattering amplitude averaged over some region in energies. Our consideration applies directly to the scattering amplitude, as follows from the discussion above and Eq.(6). Thus, we conclude that the integral in Eq.(6) is dominated by a strong scattering amplitude in the Regge kinematics. Taking into account the leading $\rho$ - meson trajectory, we obtain

$$
T(s, t)=\beta(t) \xi(t)\left(\frac{s}{s_{0}}\right)^{\alpha(t)}
$$

Here $\alpha(t)=\alpha(0)+\alpha^{\prime} t$ is the Regge trajectory, $s_{0}$ is a scale parameter, $\xi(t)$ is the signature factor

$$
\xi(t)=\frac{1-\exp [-i \pi \alpha(t)]}{\sin \pi \alpha(t)}
$$

and $\beta(t)$ stands for the reggeon residue. In what follows we adopt a model for the functional t-dependence in $\beta(t)$ obtained in dual models 13 and the quark-gluon string model [14] :

$$
\beta(t)=\frac{\beta(0)}{\Gamma[\alpha(t)]}
$$


We next calculate the integral in Eq.(6) neglecting the t-dependence of the product $\Gamma(\alpha(t)) \sin \pi \alpha(t)$

$$
\Gamma(\alpha(t)) \sin \pi \alpha(t) \simeq \Gamma(\alpha(0)) \sin \pi \alpha(0) \simeq \sqrt{\pi}
$$

and approximating $t \simeq-s / 2(1-\cos \theta)$. Then

$$
\begin{array}{r}
\int_{-1}^{1} d(\cos \theta) T(s, t)=\frac{2 \beta(0)}{\sqrt{\pi} \alpha^{\prime} s}\left(\frac{s}{s_{0}}\right)^{\alpha(0)} \\
\times\left[\frac{1-\exp \left[-\alpha^{\prime} s \ln \frac{s}{s_{0}}\right]}{\ln \frac{s}{s_{0}}}+i \frac{1-\exp \left[-\alpha^{\prime} s\left(\ln \frac{s}{s_{0}}-i \pi\right)\right]}{\ln \frac{s}{s_{0}}-i \pi}\right]
\end{array}
$$

Since the exponents are small in comparison to unity above the threshold $s=m_{D}^{2}$, we finally obtain

$$
\operatorname{Disc} M(s) \simeq \frac{i}{8 \pi} \frac{G_{F}}{\sqrt{2}} V_{c b} V_{u d}^{*} f_{\pi} f_{+}(0, s) F(s) \frac{\beta(0)}{\sqrt{\pi} \alpha^{\prime} s}\left(\frac{s}{s_{0}}\right)^{\alpha(0)} \Theta\left(s-m_{D}^{2}\right)
$$

where

$$
F(s)=\frac{1}{\ln \left(2 \alpha^{\prime} s\right)}+\frac{i}{\ln \left(2 \alpha^{\prime} s\right)-i \pi}
$$

In these formulae the intercept $\alpha(0) \simeq 0.5$ and the slope of the Regge trajectory $\alpha^{\prime} \simeq 1 \mathrm{Gev}^{-2}$. Furthermore, it is important to stress that the scale parameter $s_{0} \equiv s_{0}^{\pi D}$ is not constant, but varies with $m_{c}$. To parametrize this dependence, we will use the relation obtained in the quark gluon string model [14]

$$
s_{0}^{\pi D}=s_{0}^{\pi \pi} \frac{\bar{x}_{\pi}^{d}}{\bar{x}_{D}^{d}} \simeq\left(\alpha^{\prime}\right)^{-1} \frac{m_{c}}{2 \Lambda}
$$

where $\bar{x}_{\pi}^{d}\left(\bar{x}_{D}^{d}\right)$ is the average momentum fraction carried by the d-quark in the $\pi$ (D-) meson and $\Lambda \simeq 300-400 \mathrm{MeV}$ is the average virtuality of the light quark in the D-meson. For the physical value $m_{D} \simeq 1.9 \mathrm{GeV}$ one obtains $s_{0}^{\pi D} \simeq 2\left(\alpha^{\prime}\right)^{-1}$. It is worth noticing that the standard dual model arguments would rather suggest a universal expression for the scale factor $s_{0}^{\pi D} \simeq\left(\alpha^{\prime}\right)^{-1}$.

\section{Light cone sum rule for the $B \rightarrow D$ form factor}

Before proceeding to a calculation of the dispersion integral we need an estimate for a functional $s$ dependence of the $B \rightarrow D$ transition form factor $f_{+}(0, s)$. The idea of a method presented in this section is borrowed from a very similar problem of the pion form factor at intermediate momentum transfers, which is most conveniently solved by the light cone QCD sum rules method [15] (see also Ref. [16]). We consider the following correlation function 


$$
T_{\mu \nu}(p, k)=i \int d x e^{i k x}\left\langle D(p)\left|T\left\{\bar{c} \gamma_{\mu} b(x) \bar{b} \gamma_{\nu} \gamma_{5} d(0)\right\}\right| 0\right\rangle
$$

for Minkowskian $k^{2}=0$ and large Euclidean momentum $(p+k)^{2} \rightarrow-\infty$. The B-meson contribution to this correlation function is

$$
-2 p_{\mu} k_{\nu} i f_{B} \frac{1}{m_{B}^{2}-(p+k)^{2}} f_{+}\left(k^{2}\right)+\cdots,
$$

where ellipses stand for other Lorentz structures. On the other hand, the correlation function (12) can be calculated in QCD at large Euclidean momenta $(p+k)^{2}$. Indeed, in this case the virtuality of the heavy quark, which is of order $m_{b}^{2}-(p+k)^{2}$, is large. Thus, we can expand the heavy quark propagator in powers of slowly varying fields residing in the D-meson, which act as external fields on the propagating heavy quark. The expansion in powers of an external field is also the expansion of the propagator in powers of a deviation from the light cone $x^{2} \simeq 0$. The leading contribution is obtained by using the free heavy quark propagator in the correlation function (12). We obtain

$$
T_{\mu \nu}(p, k)=\int \frac{d^{4} x d^{4} l}{(2 \pi)^{4}\left(m_{b}^{2}-l^{2}\right)} e^{i(k-l) x}\left\langle D(p)\left|\bar{c}(x) \gamma_{\mu}\left(l_{\xi} \gamma_{\xi}+m_{b}\right) \gamma_{\nu} \gamma_{5} d(0)\right| 0\right\rangle
$$

In this formula a path-ordered gauge factor between the quark fields is implied, as required by gauge invariance. In the particular case of the Fock-Schwinger gauge $x_{\mu} A_{\mu}(x)=0$ this factor is equal to unity. We see that the answer is expressed via the one-meson matrix element of the gauge invariant non-local operator with a light-like separation $x^{2} \simeq 0$. This matrix element defines a light cone D-meson distribution amplitude. The leading twist distribution amplitude $\phi(u)$ is introduced as follows 17]:

$$
\left\langle D(p)\left|\bar{c}(x) \gamma_{\mu} \gamma_{5} d(0)\right| 0\right\rangle=-i f_{D} p_{\mu} \int_{0}^{1} d u \exp (i u p x) \phi(u)
$$

Within this definition we obtain to the leading twist accuracy (hereafter $\bar{u}=1-u$ )

$$
T_{\mu \nu}(p, k)=-i f_{D} p_{\mu} k_{\nu} \int_{0}^{1} d u \frac{\phi(u)}{m_{b}^{2}+u \bar{u} m_{D}^{2}-u(p+k)^{2}}+\cdots
$$

To match Eq.(19) with the B-meson contribution (16) to the correlation function (15), we note that Eq.(19) can be re-written as the dispersion integral with $\left(m_{b}^{2}+\right.$ $\left.u \bar{u} m_{D}^{2}\right) / u$ being the mass of an intermediate state. The duality prescription tells that this invariant mass has to be restricted from above by the duality threshold $s_{0}=\left(m_{b}+m_{0}\right)^{2}$, where $m_{0}$ does not depend on $m_{b}$. For physical value $m_{b} \simeq 4.7 \mathrm{GeV}$ one gets $s_{0} \simeq 35 \mathrm{GeV}^{2}$, thus $m_{0} \simeq 1.2 \mathrm{GeV}$. We then obtain the following sum rule

$$
\frac{2 f_{B}}{m_{B}^{2}-(p+q)^{2}} f_{+}\left(0, m_{B}\right)=f_{D} \int_{1-\frac{2 m_{0}}{m_{b}}}^{1} d u \frac{\phi(u)}{m_{b}^{2}+u \bar{u} m_{D}^{2}-u(p+q)^{2}}+\cdots
$$

To analyze a parametric behavior of the form factor $f_{+}\left(0, m_{B}\right)$, we first note that as $m_{b}^{2} \gg m_{c}^{2}$ we can neglect the second term in the denominator in the right hand side 
of Eq.(20). Next one has to distinguish between two different kinematical regimes : (i) In the limit $m_{b} \rightarrow \infty$ and $m_{c} \rightarrow 0$ D-meson becomes pion, the corresponding process being $\bar{B} \rightarrow \pi^{+} \pi^{-} \rightarrow 2 \pi^{0}$. In this case the asymptotic as $u \rightarrow 1$ behavior of $\phi(u)$ is $\phi(u) \simeq 6(1-u)$ and then

$$
f_{+}\left(0, m_{B}\right) \simeq \frac{6 f_{\pi} m_{0}^{2}}{f_{B} m_{b}^{2}} \sim m_{B}^{-3 / 2}
$$

(here we have used the fact that for $m_{Q} \rightarrow \infty f_{Q} \sim m_{Q}^{-1 / 2}$ up to logarithmical corrections.) This dependence is well known from the light cone sum rules for the $B \rightarrow \pi$ transition [16, 10].

(ii) More interesting is the case $m_{c}, m_{b} \rightarrow \infty$ with $m_{b} \gg m_{c}$. Then the D-meson distribution amplitude is strongly peaked at equal velocities of quarks, i.e. concentrated near $u=1$ [17]. We adopt the parametrization [18]

$$
\phi_{D}(u)=A_{D} \frac{u^{2} \bar{u}^{2}}{\left[\varepsilon^{2} u+\bar{u}^{2}\right]^{2}},
$$

where $\varepsilon=\Lambda /\left(\Lambda+m_{c}\right.$ ) and $A_{D}$ is fixed by the normalization ( $A_{D} \simeq 0.67$ for $m_{c} \simeq 1.3 \mathrm{GeV}$ and $\Lambda \simeq 0.33 \mathrm{GeV}$ ). Using Eqs. (21) and (23), we obtain

$$
f_{+}\left(0, m_{B}\right) \simeq \frac{4 A_{D} f_{D} m_{0}^{3}}{\left[\varepsilon^{2}+\frac{4 m_{0}^{2}}{m_{B}^{2}}\right]^{2}} \frac{1}{f_{B} m_{B}^{3}} \equiv \xi m_{B}^{-5 / 2}
$$

More faster in comparison with (22) fall off of the form factor $f_{+}\left(0, m_{B}\right)$ indicated in Eq. (24) appears quite reasonable. As $\phi_{D}(u)$ is strongly peaked near $u=1$, the integral in Eq. (21) becomes very sensitive to a change of the integration limit. Note in passing that this is precisely the reason why a naive ansatz of the type $\phi(u)=\delta\left(u-1+\Lambda / m_{c}\right)$ would produce completely wrong answer.

\section{Residue factor $\beta(0)$ and $g_{\rho D D}$ coupling constant}

In this section we obtain an estimate for the residue $\beta(0)$. To this end, we make use of the fact that a reggeon-particle vertex is an analytical continuation of the t-channnel resonance coupling constant into the region $t \sim 0$ [13. We thus consider the charge exchange process $D^{+} \pi^{-} \rightarrow D^{0} \pi^{0}$ proceeding for $t \simeq m_{\rho}^{2}$ via the $\rho$ - meson exchange. The asymptotic ( for $s \rightarrow \infty$ ) expression for the amplitude is

$$
T(s, t)=g_{\rho \pi \pi} g_{\rho D D} \frac{2 s}{t-m_{\rho}^{2}}
$$

The $\rho-\pi$ vertex is well known:

$$
g_{\rho \pi \pi} \simeq g_{\rho}, \quad\left\langle 0\left|e_{u} \bar{u} \gamma_{\mu} u+e_{d} \bar{d} \gamma_{\mu} d\right| \rho\right\rangle=\frac{m_{\rho}^{2}}{g_{\rho}} \varepsilon_{\mu},
$$


( $\varepsilon_{\mu}$ stands for the $\rho$ - meson polarization vector) and thus we have to estimate only the $\rho D$ vertex $g_{\rho D D}$. Here we again use the light cone QCD sum rules technique [9]. Consider the correlation function

$$
T_{\mu \nu}(p, q)=i \int d x e^{i p x}\left\langle\rho(q)\left|T\left\{\bar{d} \gamma_{\mu} \gamma_{5} c(x) \bar{c} \gamma_{\nu} \gamma_{5} u(0)\right\}\right| 0\right\rangle
$$

The contribution of interest is

$$
p_{\mu}\left(p_{\nu}+q_{\nu}\right)(\varepsilon p) g_{\rho D D} f_{D}^{2} \frac{1}{\left(m_{D}^{2}-p^{2}\right)\left(m_{D}^{2}-(p+q)^{2}\right)}
$$

On the other hand, we calculate this correlation function at large Euclidean momenta $p^{2},(p+q)^{2} \rightarrow-\infty$ using the Operator Product Expansion near the light cone [9, 10]. To the leading twist accuracy we obtain

$$
T_{\mu \nu}=\frac{1}{2} p_{\mu} q_{\nu}(\varepsilon p) f_{\rho} m_{\rho} \int_{0}^{1} d u \frac{g_{\perp}^{(a)}(u)-4 \Phi_{\|}(u)}{\left[m_{c}^{2}-(p+u q)^{2}\right]^{2}},
$$

where the twist 2 distribution amplitudes $g_{\perp}^{(a)}(u)$ and $\Phi_{\|}(u)$ are defined as follows 17, 19:

$$
\begin{gathered}
\left\langle\rho(q)\left|\bar{d}(x) \gamma_{\mu} u(0)\right| 0\right\rangle=q_{\mu}(\varepsilon x) f_{\rho} m_{\rho} \int_{0}^{1} d u e^{i q x} \Phi_{\|}(u) \\
+\varepsilon_{\mu} f_{\rho} m_{\rho} \int_{0}^{1} d u e^{i q x} g_{\perp}^{(v)}(u) \\
\left\langle\rho(q)\left|\bar{d}(x) \gamma_{\mu} \gamma_{5} u(0)\right| 0\right\rangle=-\frac{1}{4} f_{\rho} m_{\rho} \varepsilon_{\mu \nu \lambda \sigma} \varepsilon_{\nu} q_{\lambda} x_{\sigma} \int_{0}^{1} d u e^{i q x} g_{\perp}^{(a)}(u)
\end{gathered}
$$

To the lowest conformal spin accuracy the distribution amplitudes have very simple forms [19]:

$$
\begin{aligned}
\Phi_{\|}(u) & =i \frac{3}{2} u \bar{u}(2 u-1) \\
g_{\perp}^{(a)}(u) & =6 u \bar{u}
\end{aligned}
$$

We next use the identity $(p+u q)^{2}=\bar{u} p_{1}^{2}+u p_{2}^{2}-u \bar{u} q^{2}$ with $p_{1}^{2}=p^{2}$ and $p_{2}^{2}=(p+q)^{2}$ and the formula for the double Borel transformation in respect to $p_{1}^{2}$ and $p_{2}^{2}$

$$
B_{M_{1}^{2}, M_{2}^{2}} \frac{\Gamma(\nu)}{\left[m_{c}^{2}+u \bar{u} q^{2}-\bar{u} p_{1}^{2}-u p_{2}^{2}\right]^{\nu}}=\left(M^{2}\right)^{2-\nu} \exp \left[-\frac{m_{c}^{2}}{M^{2}}-\frac{q^{2}}{M_{1}^{2}+M_{2}^{2}}\right] \delta\left(u-u_{0}\right)
$$

where

$$
M^{2}=\frac{M_{1}^{2} M_{2}^{2}}{M_{1}^{2}+M_{2}^{2}} \quad, \quad u_{0}=\frac{M_{1}}{M_{1}^{2}+M_{2}^{2}}
$$

In a final expression we set $M_{1}^{2}=M_{2}^{2}=2 M^{2}$ and account for the fact that $\Phi_{\|}(1 / 2)=$ 0 . We then arrive at a simple sum rule

$$
g_{\rho D D} \simeq \frac{f_{\rho} m_{\rho}}{2 f_{D}^{2}} g_{\perp}^{(a)}\left(\frac{1}{2}\right)\left(\exp \left(-\frac{m_{c}^{2}}{M^{2}}-\frac{m_{\rho}^{2}}{4 m^{2}}\right)-\exp \left(-\frac{s_{0}}{M^{2}}\right)\right)
$$


Here the second exponent corresponds to a continuum subtraction with $s_{0} \simeq 6 \mathrm{GeV}^{2}$. This sum rule yields

$$
g_{\rho D D} \simeq 4
$$

On the other hand, the Regge formula taken for $t>0$ provides

$$
T\left(s, t \simeq m_{\rho}^{2}\right) \simeq \frac{\beta\left(m_{\rho}^{2}\right)}{\pi} \frac{s}{t-m_{\rho}^{2}}
$$

Comparing these expressions and taking into accout Eq.(10), we arrive at an estimate

$$
\beta(0) \simeq 2 \pi \Gamma\left[\alpha\left(m_{\rho}^{2}\right)\right] g_{\rho \pi \pi} g_{\rho D D} \simeq 140
$$

\section{$5 \quad$ Reggeon versus direct amplitude}

To obtain the amplitude of the process of interest, we write down an unsubtracted dispersion relation

$$
M(s)=\frac{1}{2 \pi i} \int_{m_{D}^{2}}^{\infty} d s^{\prime} \frac{\operatorname{Disc} M\left(s^{\prime}\right)}{s^{\prime}-s-i \varepsilon}
$$

To calculate the integral, we use a standard trick. The integral is first calculated for negative $s=-m_{B}^{2}$ and then one makes the analytical continuation $m_{B}^{2} \rightarrow$ $m_{B}^{2} \exp (-i \pi)$ to positive $s=m_{B}^{2}$. To make subsequent formulas feasible, we neglect in this calculation the s-dependence of the function $F\left(m_{B}\right)$ (see Eq. (14)). It is convenient to parametrize this quantity in the form

$$
F\left(m_{B}\right)=|F| \exp (i \phi)
$$

with $\left(a=\ln \left(2 \alpha^{\prime} m_{B}^{2}\right)\right)$

$$
\begin{aligned}
|F| & =\frac{1}{a}\left(\frac{2 a^{2}+\pi^{2}-2 \pi a}{\pi^{2}+a^{2}}\right)^{\frac{1}{2}} \simeq 0.2 \\
\phi & =\operatorname{atan} \frac{a^{2}}{a^{2}+\pi^{2}-\pi a} \simeq 50^{\circ}
\end{aligned}
$$

(here numerical values refer to the physical mass $m_{b} \simeq 4.7 \mathrm{GeV}$ ). Again, we distinguish between two different limiting cases:

(i) $m_{b} \rightarrow \infty$ and $m_{c} \rightarrow 0$. The corresponding physical process is $\bar{B} \rightarrow \pi^{+} \pi^{-} \rightarrow 2 \pi^{0}$. In this case the scale parameter is the standard $s_{0}^{\pi \pi}=\left(\alpha^{\prime}\right)^{-1}$. The asymptotic with $m_{b} \rightarrow \infty$ behavior of the $B \rightarrow \pi$ transition form factor is given (in the chiral limit) by Eq. (22). However, the asympotic estimate (22) cannot be analytically continued down to the threshold value $s=0$. To get an interpolating formula, we note that at $m_{b} \rightarrow 0 f_{+}\left(0, m_{B}\right)$ reduces to the pion electomagnetic form factor which equals 
unity at zero momentum transfer. We model this by the interpolating formula which is approximately valid for all $m_{B}$ in the dispersion integral

$$
f_{+}(0, s)=\frac{6 f_{\pi} m_{0}^{2}}{f_{B} s+6 f_{\pi} m_{0}^{2}}
$$

Neglecting for simplicity the $m_{B}$-dependence of $f_{B}$ during the calculation of the integral, we finally obtain

$$
M_{\pi \pi}^{(r)}\left(m_{B}\right)=\frac{G_{F}}{\sqrt{2}} V_{u b} V_{u d}^{*} \frac{3 \beta(0)|F|}{8 \pi \sqrt{\pi\left(\alpha^{\prime}\right)^{3}}} \frac{m_{0}^{2} f_{\pi}^{2}}{m_{B} f_{B}} e^{i\left(\phi+\frac{\pi}{4}\right)} \sim m_{B}^{-1 / 2}
$$

(here the subscript " $r$ " means the reggeon-exchange induced mechanism of weak decay.)

(ii) $m_{c}, m_{b} \rightarrow \infty$ with $m_{b} \gg m_{c}$. The corresponding physical process is $\bar{B} \rightarrow$ $D^{+} \pi^{-} \rightarrow D^{0} \pi^{0}$. Using Eqs. (15), (24) and (38), we obtain in this case

$$
M_{D \pi}^{(r)}\left(m_{B}\right)=\frac{G_{F}}{\sqrt{2}} V_{c b} V_{u d}^{*} f_{\pi} \frac{\beta(0)}{8 \pi \sqrt{\pi \alpha^{\prime} m_{c}}} \xi|F| m_{B}^{-3 / 2} e^{i(\phi+3 \pi / 4-2 \eta / \pi)}\left(1-\frac{2 \eta}{\pi}\right)
$$

In deriving this formula we have restricted ourselves by the linear order in $\eta \equiv$ $\sqrt{m_{D} / m_{B}}$. The answers we have found are to be compared with perturbative QCD amplitudes of the direct decays which are induced by the well known effective nonleptonic Hamiltonian (we display only the Cabibbo favored part)

$$
H_{e f f}=\frac{G_{F}}{\sqrt{2}} V_{c b} V_{u d}^{*}\left[C_{1}(\mu) O_{1}^{u}+C_{2}(\mu) O_{2}^{u}\right]
$$

where

$$
O_{1}^{u}=\left(\bar{c} \Gamma_{\mu} b\right)\left(\bar{d} \Gamma_{\mu} u\right) \text { and } O_{2}^{u}=\left(\bar{d} \Gamma_{\mu} b\right)\left(\bar{c} \Gamma_{\mu} u\right)
$$

The Wilson coefficients $C_{i}(\mu)$ are due to the renormalization of the bare Hamiltonian $H_{W} \sim O_{1}$ by hard gluons with virtualities larger than $\mu^{2}=O\left(m_{b}^{2}\right)$. In the leading$\log$ approximation with $\mu \simeq 5 \mathrm{GeV}, n_{f l}=5$ and $\Lambda_{\bar{M}} \simeq 200 \mathrm{MeV}$ [20]

$$
C_{1}=1.117 \text { and } C_{2}=-0.266
$$

For the $\bar{B}^{0} \rightarrow D^{0} \pi^{0}$ decay $O_{2}$ factorizes and

$$
\begin{aligned}
\left\langle D^{0} \pi^{0}\left|H_{e f f}\right| \bar{B}^{0}\right\rangle \sim & \left(C_{2}+\frac{C_{1}}{N_{c}}\right)\left\langle D\left|\bar{c} \Gamma_{\mu} u\right| 0\right\rangle\left\langle\pi\left|\bar{d} \Gamma_{\mu} b\right| B\right\rangle \\
& +C_{1}\left\langle D \pi\left|2\left(\bar{c} \Gamma_{\mu} t^{a} u\right)\left(\bar{d} \Gamma_{\mu} t^{a} b\right)\right| B\right\rangle
\end{aligned}
$$

The non-factorizable contribution to this decay which is given by the second matrix element in this formula has been estimated in Ref. [21] by the light cone QCD sum rule method. There has been found the following estimate for the ratio of the non-factorizable to the factorizable $1 / N_{c}$ amplitudes :

$$
r \equiv \frac{M^{(n f)}}{M^{(f)}} \simeq-\frac{N_{c}}{4 \pi^{2} f_{D}^{2}} \frac{p_{\alpha}\left\langle\pi\left|\bar{d} \tilde{G}_{\alpha \mu} \gamma_{\mu} \gamma_{5} b\right| B\right\rangle}{\left(m_{B}^{2}-m_{D}^{2}\right) f_{\pi}^{+}\left(m_{D}^{2}\right)} \simeq-0.7
$$


where the the $B \rightarrow \pi$ transition form factor $f_{\pi}^{+}$is defined as in Eq.(4). The direct decay amplitude is then

$$
M^{(d i r)}\left(m_{B}^{2}\right)=i \frac{G_{F}}{\sqrt{2}} V_{c b} V_{c s}^{*}\left[C_{2}+\frac{C_{1}}{N_{c}}(1+r)\right] f_{D} f_{\pi}^{+}\left(m_{D}^{2}\right)\left(m_{B}^{2}-m_{D}^{2}\right) \sim m_{B}^{1 / 2}
$$

We therefore see that the reggeon-induced amplitude is suppressed as $1 / m_{B}^{2}$ in respect to the direct one, while at $m_{B}=5.27 \mathrm{GeV}$ one obtains

$$
\frac{M_{D \pi}^{(r)}}{M_{D \pi}^{(d i r)}} \simeq-\frac{0.09}{C_{2}+\frac{C_{1}}{N_{c}}(1+r)} e^{i(\phi+\pi / 4-2 \eta / \pi)} \simeq 0.18+0.58 i
$$

The last equality in this formula is obtained with the particular value (49). Repeating the same procedure yields a similar formula for the $B \rightarrow 2 \pi^{0}$ decay. In this case (if one neglects a penguin contribution) the amplitude factorizes analogously to (48), but this time the suppression is only $1 / m_{B}$. Numerically one finds in this case

$$
\frac{M_{\pi \pi}^{(r)}}{M_{\pi \pi}^{(d i r)}} \simeq \frac{0.12}{C_{2}+\frac{C_{1}}{N_{c}}(1+r)} e^{i(\phi-\pi / 4)}
$$

where the parameter $r$ is defined analogously to Eq.(49). Our final results Eqs.(51) and (52) suggest that the rescattering amplitudes are not small and must be added to the amplitudes of direct decays. In this sense the latter can be thought of as radiative corrections to "tree" level amplitudes induced by soft FSI. We conclude that perturbative QCD most likely does not exhaust non-leptonic B-decays. The physical b-quark mass, albeit large, is still beyond the onset of the asymptotic QCD regime in nonleptonic heavy meson decays. An example of a similar interplay between soft and hard QCD is discussed in the next section.

We would like to end up this section with a comment on a relation between the direct and rescattering amplitudes. For definiteness, we will focus on the QCD sum rule approach. As has been explained in the introduction, any information about the imaginary part of the amplitude is lost there. Our method therefore yields the entire phase of the decay. The issue of the real part of the rescattering amplitude is slightly more subtle. Real parts of the amplitudes calculated in this paper correspond to the so-called "parasite" contributions to the sum rules (cf. Ref. [4]) and are due to a possibility to produce states below the B-meson pole in the corresponding channel. If the latter are omitted from an analysis of QCD sum rules (this require a careful analysis of corresponding Operator Product Expansion), then the two amplitides are simply added.

\section{Concluding remarks}

In this paper we presented a semi-quantitative calculation of FSI effects in color suppressed B-decays. Our results suggest a rather dissapointing conclusion that the 
soft physics prevents currently used methods from reaching an accuracy better than $30-50 \%$, the error is mainly due to a poor knowledge of multiparticle scattering FSI. Effects of soft FSI are as important as perturbative (and non-pertrurbative) QCD corrections up to the physical value of the b-quark mass, though they are parametrically suppressed in agreement with the color transparency (CT) picture of Bjorken [1]. In a sense the situation is reminiscent of the case of the pion electromagnetic form factor, which is dominated by the soft physics up to currently available momentum transfers $Q^{2} \simeq 10 \mathrm{GeV}^{2}$ [15]. There an additional suppression $1 / Q^{2}$ of the soft contribution overcomes the leading hard contribution due to a large numerical factor. It may yet seem surprising that in our problem perturbative QCD is insufficient even at much higher momenta $\sim m_{B}^{2}$.

A similar to our approach to the analysis of FSI effects in B-decay has been suggested in a recent paper [8] (see also [22]). We differ from Refs. [8, 22] in two aspects. We concentrate on FSI reducible to the leading reggeon exchange, while the authors of 8, 22] study worse understood elastic rescattering effects. Secondly, we calculate not only the phase but also corresponding corrections to the real parts of amplitudes. What if we apply the formulae of proceeding sections to the pomeron amplitude of elastic rescattering? We have checked that in this case we reproduce the conclusions of [8], i.e. the corresponding amplitude is not suppressed respectively to the direct one. Along with the two channel model used in [8] multiparticle contributions do not seem to cancel the parametric $m_{B}^{1 / 2}$ dependence of the pomeron amplitude. It appears therefore that the pomeron breaks down the CT picture of Ref [1]. We would like to mention, however, that assumptions underlying this conclusion are different from those done in the CT scenario, thus the final conclusion of Ref. [8] does not come too surprising. We feel that a space time picture of B-decays similar to those applied to high energy scattering would be desirable for a further study of this issue.

\section{Acknowledgments}

We would like to thank G. Eilam, A. Gotsman, M. Gronau, U. Maor and S. Nussinov for useful conversations and interest in this work. B.B. is grateful to M. Shifman for discussions. I.H. would like to especially acknowledge stimulating discussions with L. Frankfurt on a number of subjects related to this work. We are grateful to Da-Xin Zhang for bringing Ref. [22] to our attention. 


\section{References}

[1] J.D. Bjorken, Nucl. Phys. B (Proc. Suppl.) 11 (1989) 325.

[2] See e.g. L. Frankfurt, G.A. Miller and M. Strikman, Ann. Rev. Nucl. Part. Sci. 44 (1994) 501.

[3] M.J. Dugan and B. Grinstein, Phys. Lett. B255 (1991) 583.

[4] B. Blok and M.A. Shifman, Sov. J. Nucl. Phys. 45 (1987) 135, 301, 522;

B. Blok and M. Shifman, Nucl. Phys. B389 (1993) 534.

[5] M.I. Shifman, A.I. Vainshtein and V.I. Zhakharov, Nucl. Phys. B147 (1979) 385 .

[6] L. Maiani and M. Testa, Phys. Lett. B245 (1990) 585.

[7] M. Ciuchini, E. Franco, G. Martinelli and L. Silvestrini, hep-ph/9604240.

[8] J.F. Donoghue, E. Golowich, A.A. Petrov and J.M. Soares, hep-ph/9604283.

[9] V.M. Braun and I.E. Filyanov, Z. Phys. C44 (1989) 157.

[10] V. Belyaev, V. Braun, A. Khodjamirian and R. Ruckl, Phys. Rev. D51 (1995) 6177.

[11] A.V. Dobrovolskaya, A.B. Kaidalov, K.A. Ter-Martirosyan and V.R. Zoller, Phys. Lett. B229 (1989) 293.

[12] N. Isgur and M.B. Wise, Phys. Lett. B232 (1989) 113; B237 (1990) 527.

[13] See e.g. P.D.B. Collins, Introduction to Regge Theory and High Energy Physics (Cambridge Univ. Press, 1977).

[14] P.E. Volkovitsky and A.B. Kaidalov, Yad. Fiz. 35 (1982) 1231, 1556.

[15] V. Braun and I. Halperin, Phys. Lett. B328 (1994) 457.

[16] V.M. Belyaev, A.Khodjamirian and R.Ruckl, Z. Phys. C60 (1993) 349.

[17] V.L. Chernyak and A.R. Zhitnitsky, Phys. Rep. 112 (1984) 173.

[18] A. Szczepaniak, E.M. Henley and S.J. Brodsky, Phys. Lett. B243 (1990) 287.

[19] P. Ball and V.M. Braun, hep-ph/9602323.

[20] G. Altarelli and L.Maiani, Phys. Lett. B52 (1974) 352;

M.K. Gaillard and B.W. Lee, Phys. Rev. Lett. 33 (1974) 108.

[21] I. Halperin, Phys. Lett. B349 (1995) 548.

[22] H. Zheng, Phys. Lett. B356 (1995) 107. 